\title{
Conocimientos, actitudes y prácticas sobre accidentes ocupacionales en estudiantes de odontología
}

\author{
Knowledge, attitudes and practices related to occupational \\ accidents in dentistry
}

\author{
Dra. Katherine Arrieta Vergara, Dra. Shyrley Díaz Cárdenas, Dr. Farith \\ González Martínez
}

Universidad de Cartagena. Cartagena de Indias, Colombia.

\section{RESUMEN}

I ntroducción: el uso de instrumentos cortopunzantes durante la formación odontológica y la inexperiencia que tienen los estudiantes para su buen manejo incrementan el riesgo de accidentes ocupacionales.

Objetivos: describir la ocurrencia de accidentes ocupacionales en estudiantes de odontología y su relación con conocimientos, actitudes y prácticas sobre los riesgos a los que están expuestos.

Métodos: la muestra estuvo conformada por 210 estudiantes que se encontraban en prácticas preclínicas y clínicas en la Universidad de Cartagena, durante el primer periodo académico de 2011. Se utilizó una encuesta estructurada para evaluar las variables de estudio. Los datos fueron analizados a través de distribuciones de frecuencia y proporciones. Para establecer relaciones entre los conocimientos, las actitudes y las prácticas con la ocurrencia de los accidentes y las variables sociodemográficas, se utilizó la prueba Ji cuadrada con un límite de significación de 0,05 .

Resultados: los accidentes ocupacionales estuvieron presentes en 95 (45,2 \%) estudiantes, se registró un nivel bueno de conocimientos en 111 (52,8 \%) participantes, actitudes favorables en $182(86,6 \%)$ estudiantes y $190(90,4 \%)$, presentaron prácticas desfavorables; no se observaron relaciones estadísticamente significativas entre la ocurrencia de los accidentes y los conocimientos, actitudes y las practicas de los estudiantes.

Conclusiones: la ocurrencia de accidentes ocupacionales se considera alta y no se relaciona con los conocimientos, actitudes y prácticas, lo que permite suponer la necesidad de un ambiente y una cultura saludable que continuamente esté educando 
y velando por el cumplimiento de las medidas de bioseguridad desde las practicas estudiantiles.

Palabras clave: accidentes ocupacionales, conocimientos, actitudes, prácticas, exposiciones.

\section{ABSTRACT}

I ntroduction: both the use of sharp cutting instruments for dental training and the lack of experience of the students in properly handling them increase the risk of occupational accidents.

Objective: to describe the occurrence of occupational accidents in dentistry students and its relationship with the knowledge, attitudes and practices regarding the risks they are exposed to.

Methods: the sample included 210 dentistry students, who were in pre-clinical and clinical practices in the University of Cartagena, during the first academic period of 2011. A structured survey to evaluate the study variables was used. Frequency and ratio distributions served to analyze data. The Chi square test was used to establish relationships between knowledge, attitudes and practices and the prevalence of occupational accidents and the socio demographic variables, assuming a significance limit of 0,05 .

Results: ninety five students (45.2\%) suffered occupational accidents; a good level of knowledge was found in 111 students (52.8\%); favorable attitudes were seen in $182(86,6 \%)$ whereas $190(90.4 \%)$ students presented with negative practices.

There was no statistically significant relationship between the occurrence of accidents and the knowledge, attitudes and practices of the students.

Conclusions: the prevalence of occupational accidents is high, but unrelated to the knowledge, attitudes and practices, which allows assuming that healthy environment and culture are required to continue educating the students and ensuring compliance with the biosafety measures since the stage of student practices.

Key words: occupational accidents, knowledge, practices, attitudes, exposure.

\section{NTRODUCCI ÓN}

La Organización Internacional del Trabajo (OIT) y la Organización Panamericana de la Salud (OPS) estiman que cada año se producen 250 millones de accidentes laborales en todo el mundo y 3000 personas mueren cada día por causas relacionadas con el trabajo. Así, las causas de muerte vinculadas al trabajo se colocan por encima de los accidentes de tránsito, las guerras y la violencia. ${ }^{1}$

La prevención de accidentes es uno de los principales supuestos necesarios a cumplir en las actividades de riesgo, principalmente en el área de la salud, en la que muchos accidentes son causados por errores humanos, que probablemente se originan por un sistema de educación deficiente y la falta de una cultura de seguridad. ${ }^{2}$ Estudios sobre accidentes ocupacionales ocurridos en alumnos del sector de la salud demuestran que el área de odontología es más vulnerable a la presencia de estos. ${ }^{3,4}$ Los estudiantes de 
odontología están expuestos a accidentes ocupacionales durante el ejercicio de su práctica clínica en las aulas universitarias o comunitarias, particularmente al riesgo de transmisión de enfermedades infecciosas, ${ }^{5}$ por el contacto directo con líquidos potencialmente infectantes y por el uso de instrumentos de rotación y corto punzantes. ${ }^{3}$ Este riesgo se ve incrementado por la inexperiencia en el manejo de los elementos e instrumental odontológico, el escaso desarrollo de habilidades manuales y el uso no muy difundido de barreras de contención primaria. ${ }^{6}$

Conocer qué saben, cuáles son las prácticas y actitudes de los estudiantes de odontología sobre los riesgos de transmisión de enfermedades en su actividad clínica, constituye una fuente valiosa de información para el diseño de estrategias de educación y comunicación tendientes a promover en esta etapa de formación, practicas seguras con el fin de disminuir estos eventos.

El presente estudio tiene como objetivo describir la ocurrencia de accidentes ocupacionales en estudiantes de odontología y su relación con conocimientos, actitudes y prácticas sobre los riesgos a los que están expuestos.

\section{MÉTODOS}

Estudio de corte transversal, realizado en 210 estudiantes de odontología en prácticas preclínicas y clínicas en la Universidad de Cartagena, en el primer periodo académico de 2011, seleccionados a través de un muestreo probabilístico aleatorio simple. Para el cálculo del tamaño de la muestra se utilizó el programa Stata para Windows versión 10.0 ® , con una confianza del $95 \%$ y un error relativo del $2 \%$. La participación de los sujetos estuvo regulada por las normas éticas estipuladas en la declaración de Helsinki, modificación de Edimburgo 2000 y las normas científicas, técnicas y administrativas para la investigación en seres humanos; resolución 8430 de 1993 Ministerio de salud, República de Colombia.

Se aplicó una encuesta estructurada autosuministrada, que evaluaba las variables sociodemográficas (sexo y semestre), incidencia de accidentes ocupacionales, conocimientos, actitudes y prácticas, de los estudiantes con respecto a los riesgos a los que están expuestos.

La información fue organizada y depurada a través de una base de datos en Microsoft Excel ${ }^{\circledR} 2007$, luego fue analizada con el programa estadístico mencionado. Se usaron distribuciones de frecuencia y proporciones, y se tomaron intervalos de confianza del $95 \%$. Para establecer relaciones entre la ocurrencia de accidentes ocupacionales y los conocimientos, actitudes prácticas y las variables sociodemográficas se utilizó la prueba ji cuadrada con un límite de 0,05 para la significación estadística. La variable independiente fue construida a partir de dos indicadores: bueno vs escaso para conocimientos, y favorable vs desfavorable para actitudes y prácticas.

\section{RESULTADOS}

Los participantes del presente estudio tenían un promedio de edad de $21,6 \pm 2,01$ años. Del grupo encuestados $133(63,3 \%)$ correspondió al sexo femenino y $77(36,6 \%)$ al masculino. Cursaban quinto semestre $34(16,1 \%)$ estudiantes, sexto $9(4,2 \%)$, séptimo $40(19 \%)$, octavo $72(34,2 \%)$, noveno $35(16,6 \%)$ y décimo semestre $20(9,5 \%)$. 
En cuanto a la ocurrencia de accidentes, 95 estudiantes (45,2 \%) (IC $95 \%$ : 38,4$52,0)$ registraron al menos un accidente ocupacional en sus actividades prácticas clínicas o preclínicas (tabla).

Tabla. Conocimientos de los accidentes ocupacionales en los estudiantes de odontología de la Universidad de Cartagena, 2010

\begin{tabular}{|c|c|c|c|}
\hline & $\begin{array}{l}\text { Frecuencia } \\
\quad n=213\end{array}$ & $\%$ & IC $95 \%$ \\
\hline Ocurrencia de accidentes ocupacionales & 95 & 45,2 & $38,4-52,0$ \\
\hline \multicolumn{4}{|l|}{ Conocimientos } \\
\hline $\begin{array}{l}\text { Reconoce a el virus de la hepatitis B con mayor capacidad } \\
\text { infectante que el VIH }\end{array}$ & 140 & 66,6 & $58,7-74,5$ \\
\hline Identifica el mecanismo de transmisión del VHB & 134 & 64,1 & $56,0-72,2$ \\
\hline Identifica el mecanismo de transmisión del VIH & 185 & 88,1 & $83,5-92,7$ \\
\hline $\begin{array}{l}\text { Reconoce el accidente por salpicaduras como riesgo para } \\
\text { la transmisión de infecciones }\end{array}$ & 148 & 70,4 & $63,1-77,7$ \\
\hline Identifica todos los fluidos de precaución universal & 140 & 66,6 & $58,8-74,4$ \\
\hline Posee capacitación en bioseguridad & 204 & 97,1 & $94,8-99,4$ \\
\hline $\begin{array}{l}\text { Conoce de la existencia de protocolos posexposición } \\
\text { en la facultad }\end{array}$ & 205 & 97,5 & $95,2-99,8$ \\
\hline \multicolumn{4}{|l|}{ Actitudes } \\
\hline $\begin{array}{l}\text { Seguimiento de los protocolos } \\
\text { posexposición }\end{array}$ & 133 & 61,9 & $53,7-70,1$ \\
\hline Considera que se debe reenfundar las agujas (Recapping) & 140 & 66,6 & $58,9-74,3$ \\
\hline $\begin{array}{l}\text { Considera necesarias las inmunizaciones en los } \\
\text { profesionales de la salud }\end{array}$ & 190 & 90,4 & $86,3-94,5$ \\
\hline Manipulación adecuada de las quemaduras & 165 & 78,5 & $72,3-84,7$ \\
\hline \multicolumn{4}{|l|}{ Prácticas } \\
\hline $\begin{array}{l}\text { Una vez ocurrido el accidente, lo comunica y sigue el } \\
\text { protocolo posexposición }\end{array}$ & 54 & 56,8 & $43,7-69,9$ \\
\hline $\begin{array}{l}\text { Uso en todas las ocasiones de todas las barreras } \\
\text { de contención primaria }\end{array}$ & 99 & 47,3 & $37,5-57,1$ \\
\hline Elemento de protección que menos usa: gafas & 204 & 97,1 & $94,8-99,4$ \\
\hline Reenfunda las agujas luego de utilizarlas (Recapping) & 184 & 84,2 & $82,6-85,8$ \\
\hline Posee inmunizaciones contra la hepatitis B & 171 & 81,4 & $75,6-87,2$ \\
\hline Posee inmunizaciones contra el tétano & 162 & 77,1 & $70,7-83,5$ \\
\hline
\end{tabular}

Se encontró que 111 participantes (52,8 \%), tenían buen nivel de conocimientos. Con respecto a las actitudes, la mayor frecuencia fue para los valores favorables en 182 estudiantes $(86,6 \%)$ y con respecto a las prácticas, 190 estudiantes $(90,4 \%)$ incurrieron en prácticas desfavorables, no comunicaron los accidentes 40 estudiantes dañados $(43,1 \%)$ y no siguieron los protocolos posexposición 68 estudiantes $(71,5 \%)$.

Al relacionar la ocurrencia de accidentes ocupacionales con las variables sexo y semestre, se encontró relación estadísticamente significativa para el caso del semestre $(p=0,007)$, presentándose menor frecuencia de accidentes en estudiantes de quinto a séptimo semestre $33,7 \%$; en comparación con los estudiantes de octavo a décimo semestre $52,6 \%$. Sin embargo no se encontraron relaciones estadísticamente significativas entre la ocurrencia de accidentes ocupacionales y los conocimientos, actitudes y prácticas $(p>0,05)$. 


\section{DISCUSIÓN}

En este estudio la incidencia de accidentes ocupacionales fue alta, similar a la publicada por diversos autores. ${ }^{7,8}$ Sin embargo, no todos los estudiantes accidentados registraron los eventos, ni siguieron el protocolo de manejo posexposición, de hecho, existieron 40 estudiantes $(43,1 \%)$ con actitud desfavorable hacia el seguimiento de estos protocolos; esta situación coincide con lo encontrado en la literatura donde muchos estudiantes e incluso profesionales no notifican ni muestran adhesión a las recomendaciones relacionadas con la atención posexposición. ${ }^{8,9}$ La ausencia de comunicación y registro de los accidentes impide la evaluación del riesgo, la intervención oportuna y en consecuencia la prevención. Estos hallazgos demuestran la existencia de un problema que debe ser tenido en cuenta por las facultades de odontología para tomar las acciones necesarias en la implementación de todas las estrategias posibles, con el propósito de aportar ambientes seguros y adecuados en el desarrollo de las prácticas formativas que permitan la disminución de estos accidentes.

Frente al elevado número de accidentes ocurridos durante el ejercicio de las actividades clínicas, innumerables medidas de protección han sido establecidas. El uso de barreras de contención primarias es una de las medidas más utilizada por el odontólogo y su finalidad es reducir la exposición del profesional a las secreciones o fluidos corporales del paciente, su adopción debe ser realizada de forma indiscriminada con todos los sujetos y en todos los tipos de tratamientos. ${ }^{10} \mathrm{Al}$ preguntar sobre el tipo de barrera de protección personal que menos usaban durante su práctica clínica, $204(97,7 \%)$ estudiantes manifestaron que fueron las gafas, aun cuando 148 de ellos (70,4\%) conocía la existencia de riesgo de infección a través de las salpicaduras; situación que es similar a la notificada en otros estudios donde las gafas y la careta poseen los porcentajes más bajos de uso comparados con el uso de otros elementos como guantes de látex y bata. ${ }^{8,11}$

De acuerdo con los últimos informes de la Organización Mundial de la Salud (OMS), actualmente se presenta aumento de la incidencia y prevalencia de hepatitis B y C. La vacunación contra la hepatitis $B$ es un medio eficaz para disminuir el riesgo de adquisición del virus de la hepatitis B (VHB) durante las prácticas de los alumnos. ${ }^{12}$ En ese estudio se encontró que $171(81,4 \%)$ sujetos tenían experiencia acerca de la vacunación para VHB, valores similares a los encontrados por Ramos y otros, ${ }^{11}$ quienes describen inmunizaciones efectivas en el $84,1 \%$. Estos hallazgos son muy positivos frente al estudio realizado por Naidoo y otros, ${ }^{13}$ en Sudáfrica, quienes hallan que los estudiantes no tienen vacunación contra VHB; y los publicados por Raja'a Ya y otros, ${ }^{14}$ en Yemen del Sur en el 2000, quienes identifican que solo el 38,8 \% de los estudiantes están inmunizados. Sin embargo, otros estudios informan el $93 \%$ de vacunación contra el VHB. ${ }^{8}$

Dentro de los factores asociados a la ocurrencia de accidentes, se encontró relación estadísticamente significativa con el semestre $(p=0,007)$, y el grupo de octavo a décimo semestre del ciclo académico fue el que más accidentes presentó. Las causas de este resultado, posiblemente se deba a que los estudiantes de quinto a séptimo tienen menos oportunidades de contacto con instrumental causante de potenciales accidentes en razón al número de horas prácticas y por tanto, de pacientes que deben atender.

Existe una alta ocurrencia de accidentes ocupacionales en esta población a pesar de los buenos conocimientos y actitudes de los estudiantes, lo que implicaría un mayor seguimiento en las prácticas de bioseguridad y prevención de accidentes. 


\section{REFERENCI AS BI BLI OGRÁFICAS}

1. Castiglione S. Estudio sobre legislación comparada sobre riesgos del trabajo en América central [Internet]. Organización Panamericana de la Salud; 2003 [citado 29 de junio del 2011]. Disponible en: http://www. bvsde.opsoms.org/foro hispano/BVS/bvsacd/cd49/studylegisl-ca.pdf

2. Müller IC, Mastroeni MF. Tendência de acidentes em laboratórios de pesquisa. Biotecnolog Ci Desenv. 2004;33:101-8.

3. Shah T, Anwar M, Dosman J. Percutaneous injuries among dental professionals in Washington State. BMC Public Health. 2006; 6: 269.

4. Del Valle G, Castillo C, Castillo B, Bregains L, Irazuzta L, Rezzónico S, et al. Conocimientos sobre riesgos y profilaxis preventiva en estudiantes de odontología. Rev Salud Pública. 2009;2(13):32-8.

5. Tovar V, Guerra ME, Carvajal A. Accidentes laborales y riesgo de contraer infección por el virus de Inmunodeficiencia Humana y el virus de la hepatitis $\mathrm{B}$ y $\mathrm{C}$ en el consultorio odontológico. Acta Odontol Venz. 2004;42(3):218-25.

6. Klewer J, Lauscheke H, Raulinaviciute J, Sasnauskaite L, Pavilonis A, Kugler J. Assessment of HIV transmission risks in clinical situations in health care students from Germany, Lithuania and Namibia. Public Health. 2000; 115(2): 114-8.

7. Duarte R Loya, Reza S. Accidentes por Objetos Punzocortantes en Estudiantes de una Escuela de Odontología. Rev Ciencia Trabajo. 2006;21(8):131-4.

8. Hincapié A, Domínguez M, Garcés C. Conocimientos y presencia de Hepatitis B en los estudiantes de pregrado de la Facultad de Odontología de la Universidad de Antioquia en el año 2003. Rev Fac Odontol Univ Antioquía. 2004; 15(2):28-38.

9. Wicker S, Rabenau HF. Occupational exposures to bloodborne viruses among German dental professionals and students in a clinical setting. Int Arch Occup Environ Health. 2010;83(1):77-83.

10. Chagas IJ, Herdy AC, Araújo WC, Chagas MS, Orives JLB. Biossegurança: Análise e melhoria de processo em acidentes biológicos. JBC J Bras Clin Odontol Integr. 2004; 8(44): 140-3.

11. Ramos-Gomez F, Ellison J, Greenspan D, Bird W, Lowe S, Gerberding J L. Accidental exposures to blood and body fluids among care workers in dental teaching clinics: a prospective study. J Am Dent Assoc. 1997; 128: 1253-61.

12. Resende VL, Abreu MH, Paiva SM, Teixeira R, Pordeus IA. Concerns regarding hepatitis $B$ vaccination and post-vaccination test among Brazilian dentists. Virol J. 2010; 13(7): 154 .

13. Naidoo S. Dental practitioner risk, kmowledge and practice with regard to hepatitis B vaccination in South Africa. Oral Dis. 1997; 3(3):175-5.

http://scielo.sld.cu 
14. Raja'a Ya, Saeed GG, Al-Hattami AA, Al-Asadi MH, Mohammad AA, Ahmed AA, et al. Coverage and perceptions of medical sciences students towards Hepatitis $B$ virus vaccine in Sana'a City, Yemen. Saudi Med. 2002;23(10):1222-6.

Recibido: 27 de julio de 2011.

Aprobado: 24 de mayo de 2012.

Katherine M. Arrieta Vergara. Departamento de Odontología Preventiva y Social, Facultad de Odontología Universidad de Cartagena. Campus de la salud, barrio Zaragocilla. Cartagena de Indias, Colombia.

Teléf.: 095-6698173 ext. 115. Correo electrónico: katherinearrieta@hotmail.com 